\title{
THE
}

\section{Surface Activity of Poly(ethylene glycol)-Coated Silver Nanoparticles in the Presence of a Lipid Monolayer}

\author{
Nasim Ganji \\ University of Rhode Island \\ Iftheker A. Khan \\ Geoffrey D. Bothun \\ University of Rhode Island, gbothun@uri.edu
}

Follow this and additional works at: https://digitalcommons.uri.edu/che_facpubs

\section{The University of Rhode Island Faculty have made this article openly available.}

Please let us know how Open Access to this research benefits you.

This is a pre-publication author manuscript of the final, published article.

Terms of Use

This article is made available under the terms and conditions applicable towards Open Access

Policy Articles, as set forth in our Terms of Use.

\section{Citation/Publisher Attribution}

Ganji, N., Khan, I. A., \& Bothun, G. D. (2018). Surface Activity of Poly(ethylene glycol)-Coated Silver Nanoparticles in the Presence of a Lipid Monolayer. Langmuir, 34(5), 2039-2045. https://doi.org/10.1021/ acs.langmuir.7b03743

Available at: https://doi.org/10.1021/acs.langmuir.7b03743 


\title{
Surface activity of poly(ethylene glycol)-coated silver nanoparticles
}

\section{in the presence of a lipid monolayer}

Nasim Ganji, ${ }^{1}$ Iftheker A. Khan, ${ }^{1}$ Geoffrey D. Bothun ${ }^{1, *}$

Department of Chemical Engineering, University of Rhode Island, 51 Lower College Road, Kinston, RI 02881.

Corresponding author. Email: gbothun@uri.edu, Tel: +1-401-874-9518

\begin{abstract}
We have investigated the surface activity of poly(ethylene glycol) (PEG)-coated silver nanoparticles (Ag-PEG) in the presence or absence of lipid monolayers comprised of monounsaturated dioleoylphosphocholine and dioleoylphosphoglycerol (DOPC/DOPG; 1:1 mole ratio). Dynamic measurements of surface pressure demonstrated that Ag-PEG were surface-active at the air/water interface. Surface excess concentrations suggested that at high Ag-PEG subphase concentrations, Ag-PEG assembled as densely-packed monolayers in the presence and absence of a lipid monolayer. The presence of a lipid monolayer led to only a slight decrease in the excess surface concentration of Ag-PEG. Surface pressure-area isotherms showed that in the absence of lipids, Ag-PEG increased the surface pressure up to $45 \mathrm{mN} \mathrm{m}^{-1}$ upon compression before the AgPEG surface layer collapsed. Our results suggest that surface activity of Ag-PEG was due to hydrophobic interactions imparted by a combination of the amphiphilic polymer coating and the hydrophobic dodecanethiol ligands bound to the Ag-PEG surface. With lipid present, AgPEG+lipid surface pressure-area $(\pi-\mathrm{A})$ isotherms reflected Ag-PEG incorporation within the lipid monolayers. At high Ag-PEG concentrations, the $\pi-\mathrm{A}$ isotherms of the Ag-PEG+lipid films closely resembled that of Ag-PEG alone, with a minimal contribution from the lipids present.
\end{abstract}


Analysis of the subphase silver (Ag) and phosphorus (P) concentrations revealed that most of the adsorbed material remained at the air/lipid/water interface and was not forced into the aqueous subphase upon compression, confirming the presence of a composite Ag-PEG+lipid film. While interactions between 'water-soluble' nanoparticles and lipids are often considered to be dominated by electrostatic interactions, these results provide further evidence that the amphiphilic character of a nanoparticle coating can also play a significant role.

Keywords: Silver nanoparticles; PEG; surface activity; Langmuir monolayer; surface pressure

\section{Introduction}

Engineered metal NPs exhibit distinctive physicochemical properties and thus have been studied in diverse research fields such as (bio)chemical sensing, multifunctional catalysis, and drug delivery. ${ }^{1-9}$ Among them, engineered silver nanoparticles (AgNPs) are well-known for their high electrical and thermal conductivity, surface-enhanced Raman scattering, chemical stability, catalytic activity, and non-linear optical behavior; and have recently been developed for use in medical imaging and bio-sensing. ${ }^{10-12}$ The safe use of AgNPs in living systems requires evaluation of their possible cytotoxicity. Recent studies have revealed that inorganic engineered NPs (ENPs) such as silver can strongly interact with cell membranes ${ }^{13-17}$ causing cytotoxicity through a variety of disruptive mechanisms including (1) adherence of the NPs to membrane, (2) aggregation around the membrane, (3) removal of lipids from membrane, and (4) permanently embedding into membrane. ${ }^{18}$ Interfacial interactions between ENPs and cell membranes are proved to be affected by various parameters such as the physicochemical properties of the ENPs (specially surface

charge, hydrophobicity, size, shape and surface functionality), cell membrane composition, and 
the extent of exposure. ${ }^{19-21}$

Lipid monolayers or bilayers employed as model membranes can be considered a first step to investigating ENP-membrane interaction mechanisms due to their ability to mimic many relevant physicochemical features of cell membranes. ${ }^{22,23}$ Langmuir monolayers, in particular, have been used as an effective tool for characterizing specific interactions at the molecular-level with membrane-forming lipids ${ }^{12,20,24,25}$. The surface pressure-area $(\pi-A)$ isotherms of the Langmuir films reveal the intermolecular forces operating in two dimensions (2D) as well as information on the arrangement and orientation of lipids. Our previous study has addressed the effects of AgNP charge, provided by anionic and cationic polymer coatings, on the duration and extent of AgNP adsorption and the response of $\mathrm{PC} / \mathrm{PG}$ monolayers $(3: 1 \mathrm{~mol})$. Dynamic surface pressure measurements revealed that AgNP binding restructures monolayers at air/water interface, with anionic AgNPs inserting into net-anionic monolayers via hydrophobic interactions and cationic AgNP adsorbing through electrostatic attraction with PG. ${ }^{26}$

The Langmuir-Blodgett (LB) technique is further useful for studying the surface activity of amphiphilic polymer-coated NPs at interfaces that drives interfacial adsorption. Like surfactants and lipid molecules, these capped NPs self-assemble into 2D lattices at air/water interfaces. ${ }^{27,28}$ Here, we characterize the surface activity of Ag-PEG in the presence or absence of net-anionic lipid monolayers (DOPC/DOPG; 1:1 mol). PEG has been the focus of research as an effective coating material due to its biocompatibility and long period of circulation in the bloodstream. ${ }^{29-33}$ Even though PEG is completely water-soluble at room temperature, PEG of sufficiently high molecular weight can form Langmuir monolayers at the air/water interface. ${ }^{34}$ Early work analyzing 
the surface activity of PEGylated NPs has shown that the presence of PEG as a surface coating material significantly decreases interfacial tension (or increases surface pressure). Björkegren et al. ${ }^{33}$ have analyzed the surface activity of PEG-functionalized silica NPs at air/water interface and observed that NPs surface activity is proportional to the degree of NP surface functionalization. Here, we employed dynamic surface pressure measurements to evaluate the kinetics of Ag-PEG adsorption at air/water interfaces, the degree of monolayers coverage, and how the presence of lipid monolayers change these properties. Subphase silver $(\mathrm{Ag})$ and phosphorus $(\mathrm{P})$ concentrations analysis were used to confirm Ag-PEG adsorption at interface and the extent of lipid extraction.

\section{Experimental}

\subsection{Materials}

1,2-Dioleoyl-sn-glycero-3-phosphocholine (DOPC; zwitterionic lipid) and 1,2-dioleoyl-snglycero-3-phospho-(1'-rac-glycerol) (DOPG; anionic lipid) were purchased from Avanti Polar Lipids (Alabaster, AL). Figure 1A shows the chemical structure of the lipids. Chloroform $\left(\mathrm{CHCl}_{3}\right.$, $>99.8 \%)$ and 1-octanol $\left(\mathrm{CH}_{3}\left(\mathrm{CH}_{2}\right)_{7} \mathrm{OH},>99.8 \%\right)$ were purchased from Fisher Scientific (Waltham, MA). Ag-PEG dispersed in deionized (DI) water were purchased from Ocean NanoTech (San Diego, CA) and used as-received (Figure 1B). Ag-PEG were prepared by the manufacturer by coating silver NPs with organic layers consist of a monolayer of dodecanethiol, a monolayer of anionic amphiphilic polymer covalently modified with PEG. 6-Carboxyfluorescein $\left(\mathrm{C}_{21} \mathrm{H}_{12} \mathrm{O}_{7}, \sim 97 \%\right)$, nitric acid (65-71\%, TraceSELECT Ultra grad), standard silver (Ag) solution (1000 mg Ag L-1 in nitric acid, TraceCERT ${ }^{\circledR}$ grade) and standard phosphorus (P) solution (1000 mg P L ${ }^{-1}$ in $\mathrm{H}_{2} \mathrm{O}$, TraceCERT ${ }^{\circledR}$ grade) were purchased from Sigma Aldrich. Deionized (DI) ultrafiltered water for all reported measurements was obtained from a Millipore Direct-Q3 UV 
purification system (Billerica, MA) at $18.2 \mathrm{~m} \Omega$ resistance and $\mathrm{pH}$ 6.5. All materials were utilized as received.

\subsection{Nanoparticle Characterization}

NPs were characterized using transmission electron microscopy (JEOL JEM-2100F) operating at $200 \mathrm{kV}$ and Malvern Zetasizer Nano ZSX for their core radius, and hydrodynamic radius and zeta potentials, respectively. The average core radius $\left(r_{c}\right)$ of Ag-PEG was determined by analyzing multiple TEM images with the ImageJ software $(n>50) .{ }^{35}$ To measure the average zeta potentials $(\zeta)$ and hydrodynamic radius $\left(r_{h}\right)$ of Ag-PEG, the as-received particles were diluted ten-fold in deionized water and analyzed at $25^{\circ} \mathrm{C}$. The values reported are based on triplicate measurements of three different samples.

A

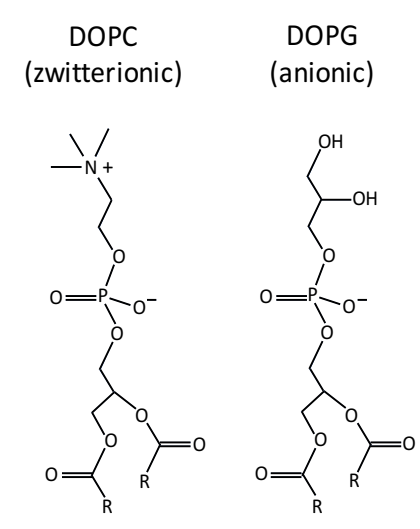

B

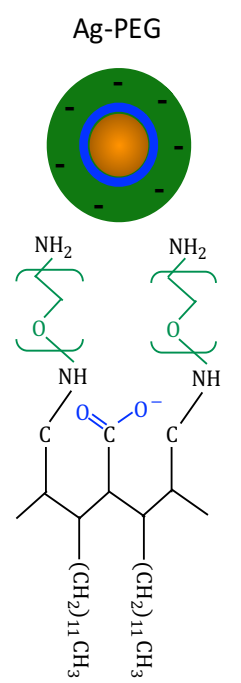

Figure 1. (A) Chemical structure of DOPC and DOPG and (B) schematic of Ag-PEG nanoparticles (not to scale). 


\subsection{Monolayer surface pressure measurements}

Surface pressures measurements were conducted using a temperature-controlled LangmuirBlodgett Teflon trough (model 102M, KSV NIMA, Biolin Scientific Inc., Linthicum Heights, MD) equipped with two symmetrically moving barriers and a paper Wilhelmy plate (KN005, KSV NIMA, Biolin Scientific Inc., Linthicum Heights, MD) as a surface pressure sensor. The trough had a fully opened area of $\sim 80 \mathrm{~cm}^{2}$ and a width of $7 \mathrm{~cm}$ (Figure 2).

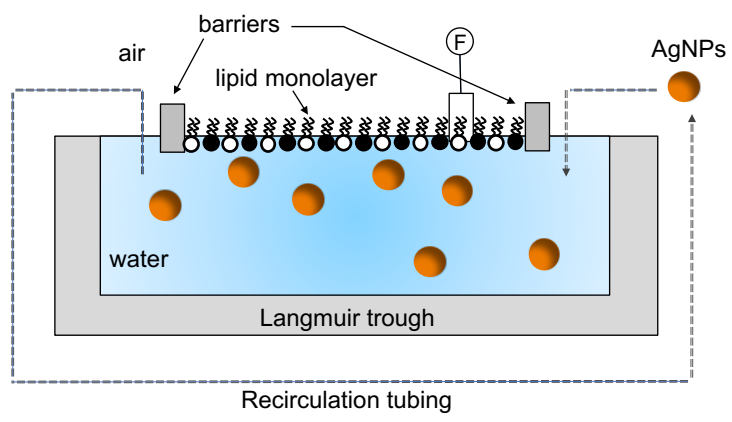

Figure 2. Schematic of the Langmuir trough system equipped with two symmetrically moving barriers, recirculation tubing and a paper Wilhelmy plate as a surface pressure sensor. The surface pressure measurements were conducted in the presence or absence of lipid monolayers and different bulk concentrations of nanoparticles.

The experiments were conducted at $25{ }^{\circ} \mathrm{C}$ through the following steps. (1) The trough and barriers were cleaned thoroughly with chloroform and then ethanol, followed by rinsing with DI water. (2) The trough was then filled with DI water and the Wilhelmy plate was equilibrated in the subphase. (3) The water surface was cleaned through compression/aspiration/expansion cycles, and followed by spreading an aliquot of dissolved lipid (DOPC/DOPG; 1:1 mole ratio) in chloroform (1 mM) on the air/water interface. The water subphase volume within the trough was $140 \mathrm{~mL}$ and approximately $9 \times 10^{15}$ lipid molecules were spread at the air/water interface. (4) The solvent was 
allowed to evaporate for $45 \mathrm{~min}$ and the monolayers were compressed and expanded at a constant barriers rate $10 \mathrm{~cm}^{2} \mathrm{~min}^{-1}$ to obtain the surface pressure-area $(\pi-A)$ isotherms. (5) After recording compression/expansion isotherms, Ag-PEG was injected into the subphase without disturbing the monolayer and dynamic changes in surface pressure $(\Delta \pi)$ were monitored for $160 \mathrm{~min}$. (6) The monolayers were then subjected to an additional compression/expansion cycle. The dynamic changes in surface pressure $(\Delta \pi-t)$ and surface pressure-area $(\pi-A)$ isotherms were recorded at different amounts of Ag-PEG loaded in the subphase $(5,25,50,100,300$, and $500 \mu \mathrm{L})$.

The compression/expansion rate applied for all runs was $10 \mathrm{~cm}^{2} \mathrm{~min}^{-1}$, which corresponded to an area deformation rate, $d\left(\Delta A / A_{0}\right) / d t$, of about $2 \times 10^{-3} \mathrm{~s}^{-1}$. The total area of the trough during the cycles ranged roughly from $20-70 \mathrm{~cm}^{2}$. In all isotherms experiments, at least three consecutive cycles were performed and the ones in which the shape of the $(\pi-A)$ curves remained constant were analysed and are presented here. Ag-PEG were mixed within the subphase by recycling the solution using a peristaltic pump at a flowrate of $0.8 \mathrm{~mL} \mathrm{~s}^{-1}$. The same monolayer experiments were conducted as mentioned above but in the absence of lipid monolayers to determine the surface activity and adsorption kinetics of Ag-PEG alone. All experiments were conducted at least in duplicate. Sample volumes of $2 \mathrm{~mL}$ were removed from the Langmuir trough subphase at the end of monolayer experiments for analysis of silver $(\mathrm{Ag})$ and phosphorus $(\mathrm{P})$ concentrations in the subphase.

\subsection{Subphase analysis of NPs and lipid (phosphorus) concentration}

Ultraviolet-visible spectroscopy (UV-vis, model: Cary 50, Varian, Palo Alto, CA) and inductively coupled plasma mass spectroscopy (ICP-MS, model: iCAP Q, Thermo Scientific, 
Waltham, MA) were used to measure the subphase concentrations of Ag and P, respectively. For [Ag] determined by UV-vis spectroscopy, plasmon resonance absorption was measured based on the maximum peak height at wavelengths of $425 \mathrm{~nm}$ after baseline subtraction. For [P] determined by ICP-MS, samples were digested using nitric acid $(200 \mu \mathrm{L})$ and then diluted 10-fold with DI water. Standard solutions containing different concentration $\left(0.1,1,10,100\right.$ and $\left.1000 \mu \mathrm{g} \mathrm{L}^{-1}\right)$ of phosphorus were used for instrument calibration. Trace amounts of $\mathrm{P}$ measured in deionized water and digestion acid solution were subtracted from the reported values. All measurements were conducted in triplicate.

\section{Results and Discussion}

\subsection{Ag-PEG characterization}

Ag-PEG nanoparticles were characterized prior to the monolayer experiments for their size, zeta potentials, stability and extent of dissolution. As shown in Figure 3A, the average core radius $\left(r_{c}\right)$ was $6 \pm 2 \mathrm{~nm}$ based on analysis of TEM images. The polymer coatings surrounding Ag-PEG were not observed in the micrographs. The mean hydrodynamic radius $\left(r_{h}\right)$ and zeta potential $(\zeta)$ were measured to be $15 \pm 2 \mathrm{~nm}(0.04$ polydispersity index $)$ and $-10.6 \pm 0.1 \mathrm{mV}$, respectively. The average coating thickness based on the difference between $r_{h}$ and $r_{c}$ was $9 \mathrm{~nm}$. The maximum surface plasmon resonance (SPR) absorbance was observed at a wavelength of $425 \mathrm{~nm}$ (Figure 3B). Ag-PEG SPR absorbance was measured by UV-vis spectroscopy over 3 months, and there was no significant shift and reduction in the SPR wavelength indicating that the nanoparticles were stable. Similar to our previous study on anionic $(\mathrm{COOH})$ and cationic $(\mathrm{NH})$-coated $\mathrm{AgNPs},{ }^{26}$ considering that the monolayer experiments were conducted within 3 months of receiving the samples, we did not account for NP dissolution in our analysis. 

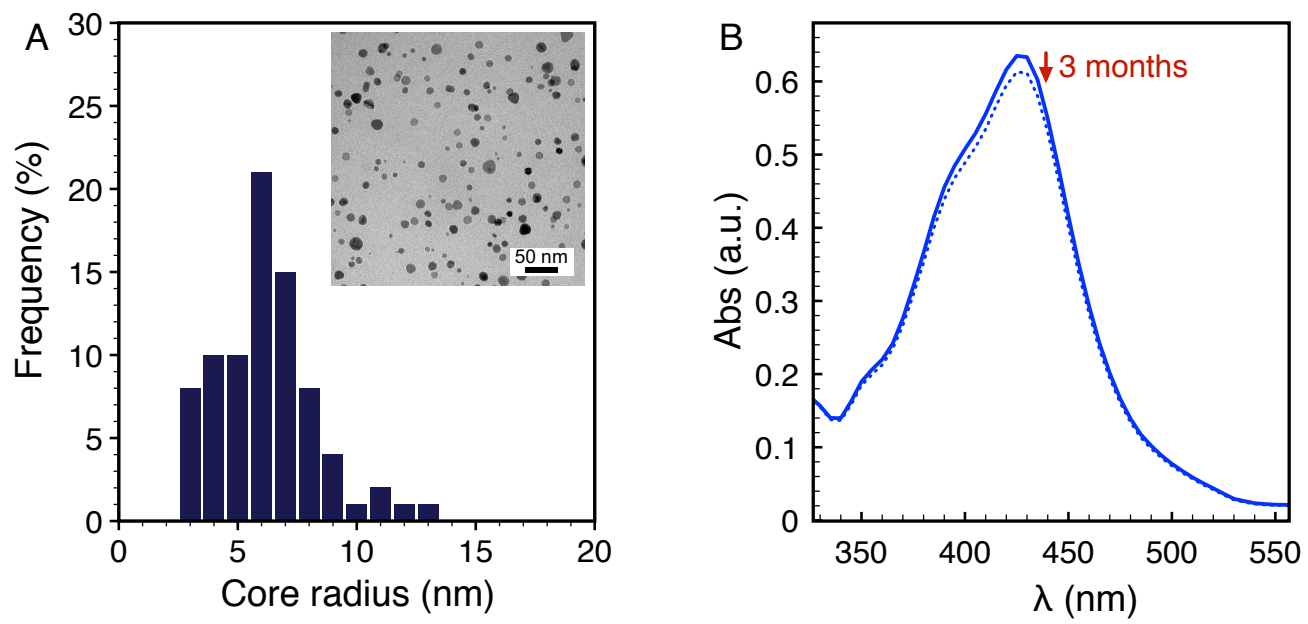

Figure 3. (A) Histogram plot of AgPEG NPs core radius $\left(r_{c}\right)$, based on TEM analysis (inset: representative micrograph). The core radius $\left(r_{c}\right)$ of the NPs was determined by analyzing TEM images with the ImageJ software (n > 50); (B) UV-vis spectra of AgPEG NPs over 3 months.

\subsection{Dynamic surface pressure measurements}

The adsorption of Ag-PEG was first examined at air/water and air/lipid/water interfaces at AgPEG concentrations from 0.04 to $3.55 \mathrm{mg} \mathrm{L}^{-1}$. Dynamic changes in surface pressure, $\Delta \pi$, were determined as $\Delta \pi=\pi(t)-\pi_{i}$, where $\pi(t)$ is the dynamic surface pressure after NPs injection and $\pi_{i}$ is the initial surface pressure of the air/water $\left(\pi_{i}=0\right.$ where $\gamma_{i}=\gamma_{0} \approx 70 \mathrm{mN} \mathrm{m}^{-1}$; Figure 4A) or air/lipid/water $\left(\pi_{i}=10\right.$ where $\gamma_{i}=\gamma_{L} \approx 60 \mathrm{mN} \mathrm{m}^{-1}$; Figure 4B) interfaces.

As shown in Figure 4C, Ag-PEG were surface active as observed by the increase in surface pressure $(\Delta \pi)$ over 160 min with increasing Ag-PEG concentration. The surface activity can be attributed to the PEGylated amphiphilic polymer coating. At high concentrations ([AgPEG] $\geq$ $0.71 \mathrm{mg} \mathrm{L}^{-1}$ ), the long term gradual increase in $\Delta \pi$ after the initial adsorption (up to $30 \mathrm{~min}$ ) 
suggests that there may have been an adsorption barrier that limited Ag-PEG adsorption. This barrier may have been due to high surface pressures, or fewer 'vacant sites' available for Ag-PEG adsorption, where Ag-PEG diffused back into the bulk phase and increased the timescale of the dynamic surface pressure changes. ${ }^{36,37}$ Björkegren et al. have reported similar results for PEGylated silica NPs surface activity at air/water interface. ${ }^{33}$

In the presence of DOPC/DOPG monolayers at an initial surface pressure of $10 \mathrm{mN} \mathrm{m}^{-1}$ (Figure 4B) Ag-PEG remained surface activity and the lipid monolayer did not prevent Ag-PEG adsorption at the interface (Figure 4D). Considering that both Ag-PEG and DOPC/DOPG monolayers exhibit a net negative charge, adsorption can be attributed to hydrophobic interactions. Xi et al. ${ }^{21}$ have also demonstrated that Ag-PEG similar to those used in this study bind to DOPC/DOPG bilayer vesicles. In their work, it was proposed that the surface activity of the PEG-polymer coating may have facilitated membrane penetration through hydrophobic interactions despite electrostatic repulsion. 
A
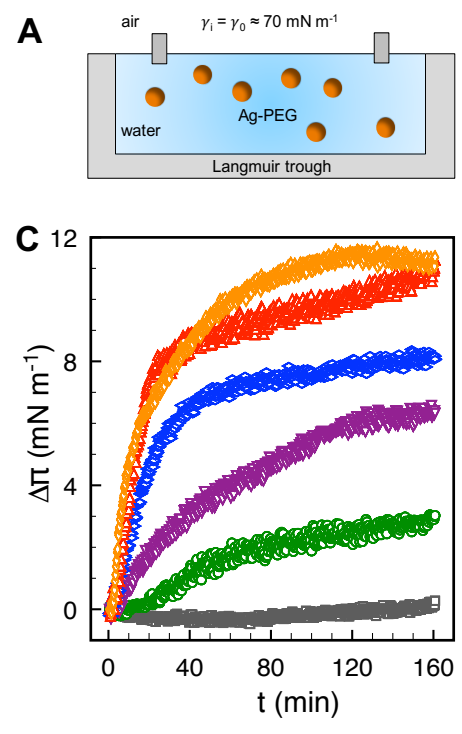

E $\quad[\mathrm{Ag}-\mathrm{PEG}]_{\mathrm{eq}}\left(10^{-7} \mathrm{~mol} \mathrm{~m}^{-3}\right)$

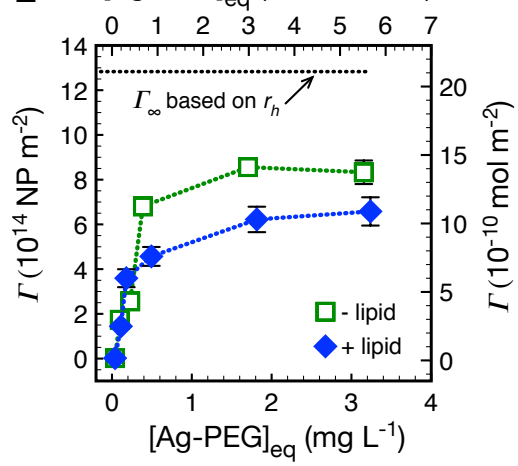

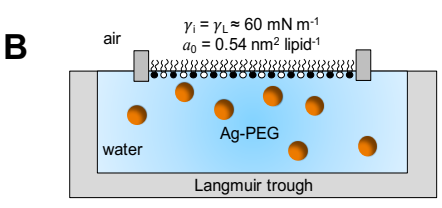

D 12

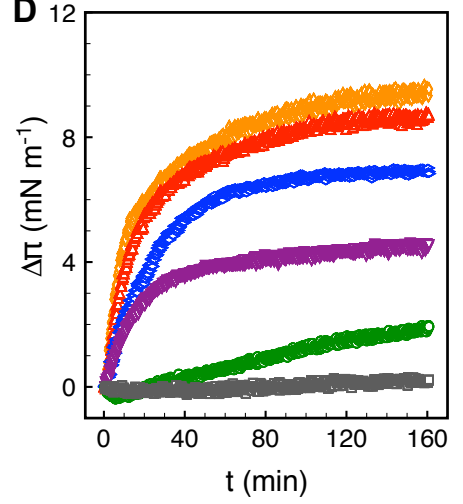

Ag-PEG $\left(\mathrm{mg} \mathrm{L}^{-1}\right)$

$\rightarrow-3.55$

$-\leftarrow 2.11$

$\multimap 0.71$

$\rightarrow-0.35$

$\rightarrow 0.18$

$-\square-0.04$

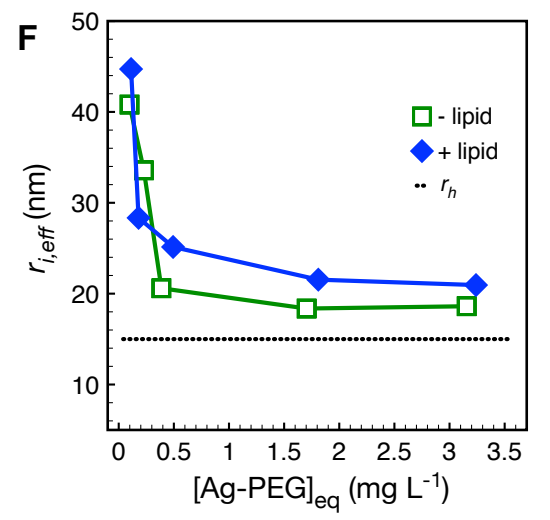

Figure 4. Schematic of Ag-PEG adsorption at (A) air/water interface $\left(\pi_{i}=0\right.$ where $\gamma_{i}=\gamma_{0} \approx 70$ $\mathrm{mN} \mathrm{m}^{-1}$ ) and (B) air/lipid/water interfaces $\left(\pi_{i}=10\right.$ where $\left.\gamma_{i}=\gamma_{L} \approx 60 \mathrm{mN} \mathrm{m}^{-1}\right)$. Dynamic changes in surface pressure $(\Delta \pi-t)$ are shown after Ag-PEG injection in (C) the absence and (D) the presence of DOPC/DOPG monolayers. (E) Excess Ag-PEG surface concentrations $\left(\Gamma, \mathrm{NP} \mathrm{m}^{2}\right.$ or mol $\mathrm{m}^{2}$ ) as a function of the equilibrium Ag-PEG concentration and (F) the resulting effective interface radius $\left(r_{i, e f f}\right)$ of Ag-PEG calculated assuming 2D hexagonal packing.

The dynamic surface pressure measurements show that Ag-PEG are surface active in the absence and presence of the lipid monolayer. However, greater surface activity was observed at the air/water interface, which suggests that the presence of a lipid monolayer may have reduced Ag- 
PEG adsorption or that Ag-PEG may have removed lipids from the interface. To address this, the subphase concentrations of Ag-PEG and phosphorus [P] were analyzed by UV-vis spectroscopy and ICP-MS, respectively. In the case of Ag-PEG, the excess surface concentration, $\Gamma$, was determined by mass balance as $\Gamma=\left(c_{i}-c_{e q}\right)_{b} V\left(V_{N P} \rho_{A g} A\right)^{-1}$ where $\left(c_{i}-c_{e q}\right)_{b}$ is the change in bulk Ag-PEG concentration from initial $\left(c_{i}\right)$ to pseudo-equilibrium $\left(c_{e q}\right), V_{N P}$ is the mean $\mathrm{Ag}$ PEG volume based on $r_{c}, \rho_{A g}$ is the density of silver, and $V$ and $A$ are the trough volume and area, respectively. The maximum surface concentration, $\Gamma_{\infty}$, was determined as $\Gamma_{\infty}=0.9069 A_{N P}^{-1}$ where 0.9069 is the 2D hexagonal packing density of spheres and $A_{N P}$ is the cross-sectional area of Ag-PEG based on the mean hydrodynamic radius, $r_{h}$. The effective interface radius of the nanoparticles $\left(r_{i, e f f}\right)$ were also calculated based on excess Ag-PEG surface concentration and assuming 2D hexagonal packing of spherical NPs (Figure 4F).

Excess Ag-PEG surface concentrations are shown in Figure 4E as a function of the equilibrium Ag-PEG concentration, $[\mathrm{Ag}-\mathrm{PEG}]_{\mathrm{eq}}=c_{\text {eq }}$. Based on $\Gamma$, greater Ag-PEG adsorption was observed in the absence of a lipid monolayer. The reduction in Ag-PEG adsorption when a lipid monolayer was present ranged from 21 to $33 \%$ at high Ag-PEG concentrations $\left(\geq 0.71 \mathrm{mg} \mathrm{L}^{-1}\right)$. However, the extent of Ag-PEG adsorption in the presence of a lipid monolayer suggests that Ag-PEG did not simply displace the lipids from the interface or that the lipids packed more tightly at the interface to accommodate Ag-PEG (which would have led to a significant increase in surface pressure). This was confirmed by the subphase phosphorous concentration (each lipid molecule contains a single $\mathrm{P}$ atom). Values for $[\mathrm{P}]$ in the bulk in the absence and presence of a lipid monolayer were similar, suggesting that lipid extraction was not a significant factor (Figure 5). Therefore, we 
conclude that Ag-PEG did not extract lipids from the monolayers and that the lipids remained at the interface to form a mixed Ag-PEG+lipid film.

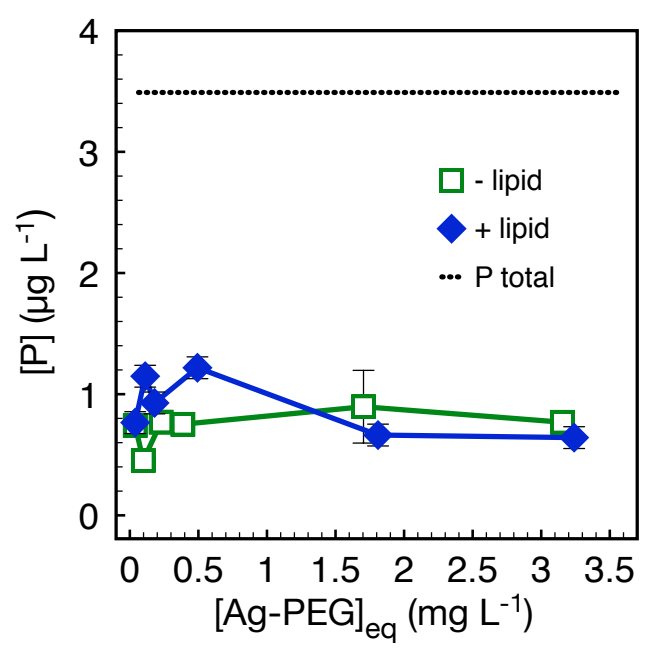

Figure 5. Subphase phosphorus concentration determined by ICP-MS (note that each lipid molecule contains a single $\mathrm{P}$ atom in the headgroup). Error bars represent standard deviation from average value. P total reflects the total amount of lipid added to the air/water interface.

\subsection{Monolayer $\pi-A$ isotherms}

Results from dynamic surface pressure show that Ag-PEG and lipid + Ag-PEG monolayers form at low surface pressures. Surface pressure-area $(\pi-\mathrm{A})$ isotherms were measured to determine the stability of these films and, in the case of lipid + Ag-PEG monolayers, to determine if the ability to lower interfacial tension (or raise $\pi$ ) is additive. Isotherms for Ag-PEG, lipid, and Ag-PEG+lipid are shown in Figure 6. For Ag-PEG, a dense monolayer was formed at $[\mathrm{Ag}-\mathrm{PEG}] \geq 0.71 \mathrm{mg} \mathrm{L}{ }^{-1}$ as demonstrated by the high surface pressure (up to $45 \mathrm{mN} \mathrm{m}^{-1}$ upon compression), monolayer collapse ${ }^{38}$, and hysteresis ${ }^{39}$ upon expansion. These features were not observed at lower [Ag-PEG], suggesting that there was no aggregation or entanglement at the interface and Ag-PEG formed stable monolayers with reversible compression behavior. 

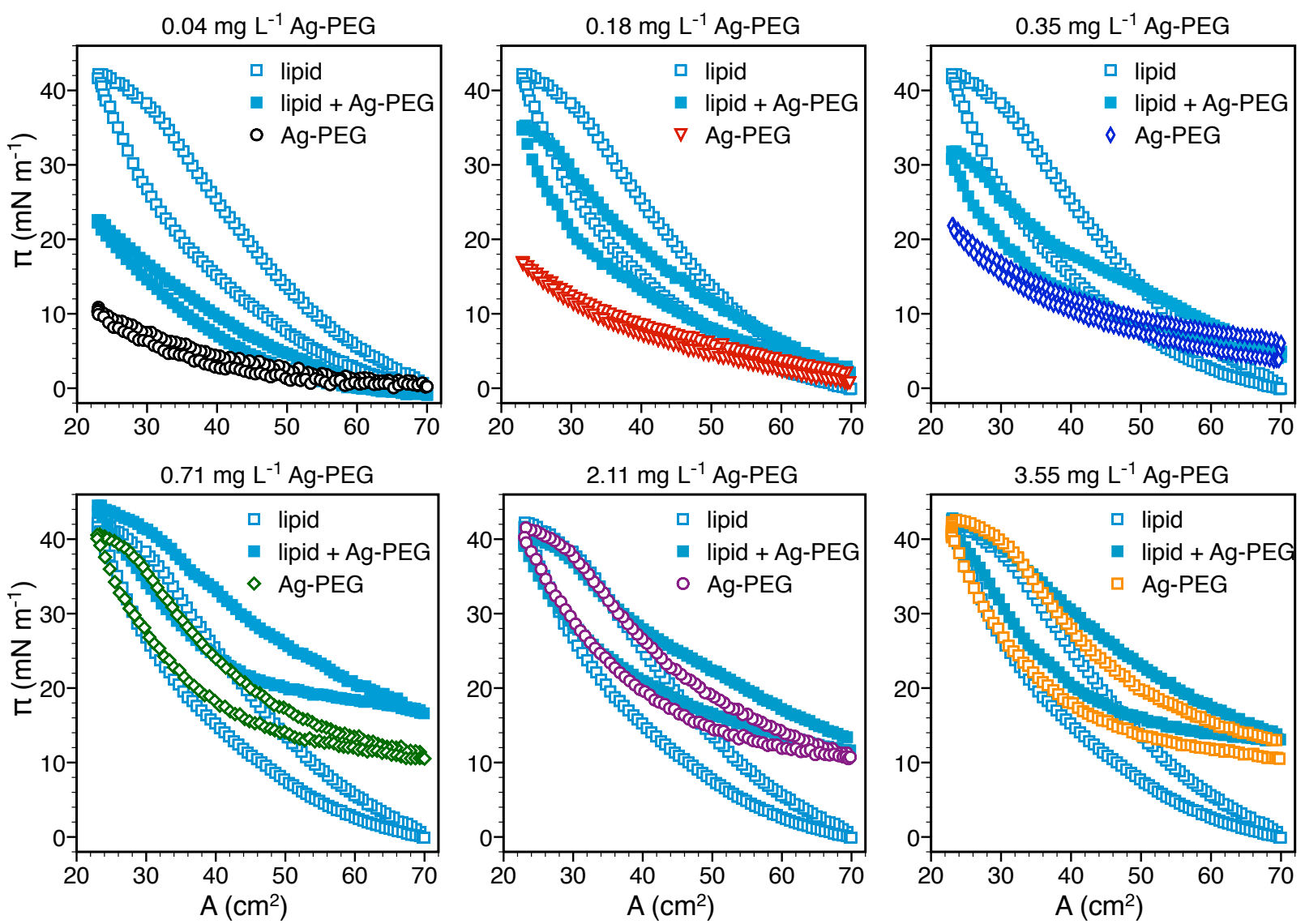

Figure 6. A comparison between compression-expansion isotherms of Ag-PEG NPs at air/water and air/lipid/water interface, at Ag-PEG concentrations from 0.04 to $3.55 \mathrm{mg} \mathrm{L}^{-1}$ (the compression is the higher curve, and the expansion is the lower curve in each case).

The collapse pressure $\left(\pi_{c}, \mathrm{mN} \mathrm{m}^{-1}\right)$ and collapse area $\left(A_{c}, \mathrm{~cm}^{2}\right)$ were determined from $\pi-\mathrm{A}$ isotherms of Ag-PEG at high nanoparticle concentrations (0.71 to $\left.3.55 \mathrm{mg} \mathrm{L}^{-1}\right)$ (Figure 7). The collapse pressure was directly proportional to Ag-PEG concentration. Based on $A_{\mathfrak{c}}$, and assuming 2D hexagonal packing, an effective Ag-PEG radius of $12.5 \pm 3.9 \mathrm{~nm}$ was calculated at the interface. The calculated 'interface radius' of the nanoparticles is consistent with the measured hydrodynamic radius. Hence, Ag-PEG assembled as densely packed monolayers at the air/water 
interface at high concentrations, and the monolayers collapsed once they exceeded hexagonal packing.

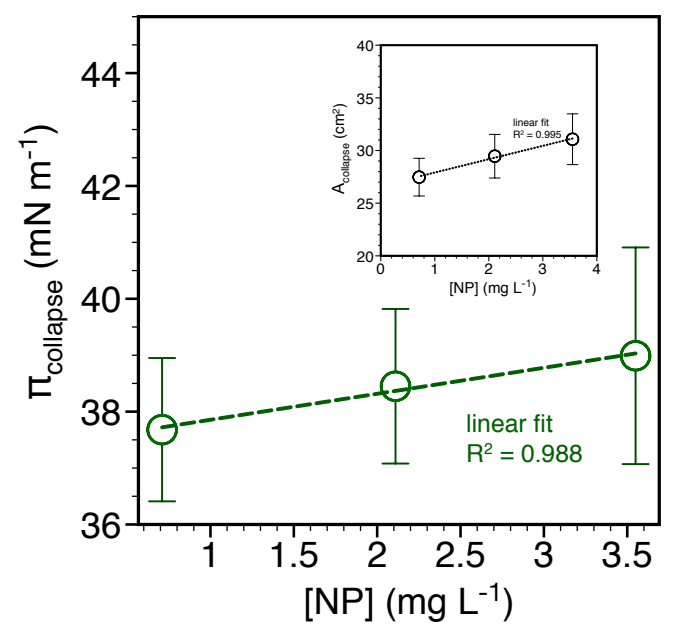

Figure 7. The collapse pressure $\left(\pi_{c}, \mathrm{mN} \mathrm{m}^{-1}\right)$ and collapse area $\left(A_{\mathrm{c}}, \mathrm{cm}^{2}\right)$ form $\pi$-A isotherms of Ag-PEG at high nanoparticle concentrations ( 0.71 to $\left.3.55 \mathrm{mg} \mathrm{L}^{-1}\right)$.

A comparison between compression/expansion isotherms of Ag-PEG at air/water and air/lipid/water interfaces are shown in Figure 6. At low Ag-PEG concentrations ([AgPEG] $\leq 0.35$ $\mathrm{mg} \mathrm{L}^{-1}$ ), the isotherm shifted to smaller area with respect to the isotherm of the lipid mixture alone, noting that more compression was necessary for the Ag-PEG+lipid films to attain the same arbitrary surface pressure compared to pure lipid film. This behavior is not attributed to the extraction of lipid molecules (Figure 5), but rather lipid adsorption onto Ag-PEG at the air/water interface that rendered the Ag-PEG more hydrophobic and reduced the effective lipid surface concentration for lowering interfacial tension (i.e. a 'subtractive' effect). At higher Ag-PEG concentrations $\left([\mathrm{AgPEG}] \geq 0.71 \mathrm{mg} \mathrm{L}^{-1}\right)$ the surface pressure of mixed Ag-PEG+lipid films were greater than the individual components at low surface areas, denoting an 'additive effect.' This apparent additive effect was not observed at high surface pressures where the $\pi-\mathrm{A}$ isotherms more closely resembled that for Ag-PEG than for lipids. At these conditions the isotherms were also 
reversible, noting that upon compression Ag-PEG did not squeeze out or escape into the subphase. Therefore, we concluded that in presence of lipids, there was a composite Ag-PEG+lipid film at the interface, where most of the adsorbed entities remained at the air/water interface.

\section{Conclusion}

Previous studies have demonstrated that NP-lipid monolayer and lipid bilayer interactions are not only governed by electrostatic interactions, and our work highlights the role of hydrophobic interactions in NP adsorption or penetration into net anionic lipid monolayers, where electrostatic repulsion between anionic NPs and anionic DOPG lipids hinder NP adsorption. Our results show that surface active Ag-PEG can adhere to and perturb net anionic lipid monolayers. Hydrophobic interactions appear to be a main driving force for Ag-PEG adsorption, where the presence of an anionic lipid appears to play a minimal role in reducing Ag-PEG adsorption to the interface. Furthermore, noting that upon compression Ag-PEG do not squeeze out or escape into the subphase, we conclude that in presence of lipids there is a composite Ag-PEG+lipid film at the interface. In these films, the adsorbed material remains at the interface. Ag-PEG likely cover themselves with lipids in a self-assembly process, thus becoming an integral part of the interfacial film. This finding is in agreement with recent studies where it has been shown that hydrophobic NPs can be encapsulated by a surfactant lipoprotein corona and trapped at the surfactant monolayer upon compression. ${ }^{40}$

\section{Acknowledgments}

This material is based upon work supported by the National Science Foundation (NSF) under Grant No. CBET-1055652. Additional resources were provided by the Rhode Island Center for 
Nanoscience and Nanotechnology, which is supported by RI NSF EPSCoR under Grant No. OIA1655221. We thank Professor Katie Kelly in the Graduate School of Oceanography at URI for assisting in ICP-MS measurements.

\section{References}

(1) Chen, K. L.; Bothun, G. D. Nanoparticles Meet Cell Membranes: Probing Nonspecific Interactions Using Model Membranes. Environ. Sci. Technol. 2014, 48 (2), 873-880.

(2) Zupanc, J. A New Approach to Analyse Effects of Nanoparticles on Lipid Vesicles. Int. J. Biomed. Nanosci. Nanotechnol. 2010, 1 (1), 34-51.

(3) Melby, E. S.; Mensch, A. C.; Lohse, S. E.; Hu, D.; Orr, G.; Murphy, C. J.; Hamers, R. J.; Pedersen, J. a. Formation of Supported Lipid Bilayers Containing Phase-Segregated Domains and Their Interaction with Gold Nanoparticles. Environ. Sci. Nano 2015, 3 (1), DOI: 10.1039/C5EN00098J.

(4) Chen, L.; Deming, C. P.; Peng, Y.; Hu, P.; Stofan, J.; Chen, S. Gold Core@silver Semishell Janus Nanoparticles Prepared by Interfacial Etching. Nanoscale 2016, 8 (30), 14565-14572.

(5) Khan, Z.; Al-Thabaiti, S. A.; Al-Nowaiser, F. M.; Obaid, A. Y.; Al-Youbi, A. O.; Malik, M. A. Kinetics of Silver Nanoparticle Growth in Aqueous Polymer Solutions. 1st Nano Update. Arab. J. Chem. 2012, 5 (4), $453-459$.

(6) K. Lance Kelly, Eduardo Coronado, Lin Lin Zhao, and G. C. S. The Optical Properties of Metal Nanoparticles- The Influence of Size, Shape, and Dielectric Environment. J. Phys. Chem. B 2003, 107 (3), 668-677.

(7) Mahato, M.; Sarkar, R.; Pal, P.; Talapatra, G. B. Formation of Silver Nanoparticle at Phospholipid Template Using Langmuir-Blodgett Technique and Its Surface-Enhanced Raman Spectroscopy Application. Indian J. Phys. 2015, 89 (10), 997-1005.

(8) Sun, Y.; Xia, Y. Shape-Controlled Synthesis of Gold and Silver Nanoparticles Author ( S ): Yugang Sun and Younan Xia Published by : American Association for the Advancement of Science Stable URL : http://www.jstor.org/stable/3833065 . Am. Assoc. Adv. Sci. 2011, 298 (5601), 2176-2179.

(9) Abbasi, A.; Park, K.; Bose, A.; Bothun, G. D. Near-Infrared Responsive Gold-Layersome Nanoshells. 
Langmuir 2017, 33 (21), 5321-5327.

(10) Prabhu, S.; Poulose, E. K. Silver Nanoparticles: Mechanism of Antimicrobial Action, Synthesis, Medical Applications, and Toxicity Effects. Int. Nano Lett. 2012, 2 (1), 32.

(11) Tran, Q. H.; Nguyen, V. Q.; Le, A.-T. Silver Nanoparticles: Synthesis, Properties, Toxicology, Applications and Perspectives. Adv. Nat. Sci. Nanosci. Nanotechnol. 2013, 4 (3), 33001.

(12) Wang, Q.; Lim, M.; Liu, X.; Wang, Z.; Chen, K. L. Influence of Solution Chemistry and Soft Protein Coronas on the Interactions of Silver Nanoparticles with Model Biological Membranes. Environ. Sci. Technol. 2016, 50 (5), 2301-2309.

(13) Johnston, H.; Pojana, G.; Zuin, S.; Jacobsen, N. R.; Møller, P.; Loft, S.; Semmler-Behnke, M.; McGuiness, C.; Balharry, D.; Marcomini, A.; et al. Engineered Nanomaterial Risk. Lessons Learnt from Completed Nanotoxicology Studies: Potential Solutions to Current and Future Challenges. Crit. Rev. Toxicol. 2013, 43 (1), 1-20.

(14) Broda, J.; Setzler, J.; Leifert, A.; Steitz, J.; Benz, R.; Simon, U.; Wenzel, W. Ligand-Lipid and Ligand-Core Affinity Control the Interaction of Gold Nanoparticles with Artificial Lipid Bilayers and Cell Membranes. Nanomedicine 2016, 12 (5), 1409-1419.

(15) Laurencin, M.; Georgelin, T.; Malezieux, B.; Siaugue, J. M.; Ménager, C. Interactions between Giant Unilamellar Vesicles and Charged Core-Shell Magnetic Nanoparticles. Langmuir 2010, 26 (20), 1602516030.

(16) Chen, K. L.; Bothun, G. D. Nanoparticles Meet Cell Membranes: Probing Nonspecific Interactions Using Model Membranes. Environ. Sci. Technol. 2014, 48 (2), 873-880.

(17) Nel, A. E.; Mädler, L.; Velegol, D.; Xia, T.; Hoek, E. M. V; Somasundaran, P.; Klaessig, F.; Castranova, V.; Thompson, M. Understanding Biophysicochemical Interactions at the Nano-Bio Interface. Nat. Mater. 2009, $8(7), 543-557$.

(18) Goreham, R. V.; Thompson, V. C.; Samura, Y.; Gibson, C. T.; Shapter, J. G.; Köper, I. Interaction of Silver Nanoparticles with Tethered Bilayer Lipid Membranes. Langmuir 2015, 31, 5868-5874.

(19) Forest, V.; Cottier, M.; Pourchez, J. Electrostatic Interactions Favor the Binding of Positive Nanoparticles on Cells: A Reductive Theory. Nano Today 2015, 10 (6), 677-680.

(20) Torrano, A. A.; Pereira, Â. S.; Oliveira, O. N.; Barros-Timmons, A. Probing the Interaction of Oppositely 
Charged Gold Nanoparticles with DPPG and DPPC Langmuir Monolayers as Cell Membrane Models. Colloids Surfaces B Biointerfaces 2013, 108, 120-126.

(21) Xi, A.; Bothun, G. D. Centrifugation-Based Assay for Examining Nanoparticle-Lipid Membrane Binding and Disruption. Analyst 2014, 139 (5), 973-981.

(22) Guzmán, E.; Santini, E.; Ferrari, M.; Liggieri, L.; Ravera, F. Interfacial Properties of Mixed DPPCHydrophobic Fumed Silica Nanoparticle Layers. J. Phys. Chem. C 2015, 119 (36), 21024-21034.

(23) Peetla, C.; Stine, a; Labhasetwar, V. Biophysical Interactions with Model Lipid Membranes: Applications in Drug Discovery and Drug Delivery. Mol. Pharm. 2009, 6 (5), 1264-1276. Gyulai, G.; Pénzes, C. B.; Mohaib, M.; Csempesz, F.; Kiss, E. Influence of Surface Properties of Polymeric Nanoparticles on Their Membrane Affinity. Eur. Polym. J. 2013, 49, 2495-2503.

Guzmán, E.; Ferrari, M.; Santini, E.; Liggieri, L.; Ravera, F. Colloids and Surfaces B : Biointerfaces Effect of Silica Nanoparticles on the Interfacial Properties of a Canonical Lipid Mixture. 2015, 136, 971-980.

(26) BOTHUN, G. D.; Ganji, N.; Khan, I.; Xi, A.; Bobba, C. Anionic and Cationic Silver Nanoparticle Binding Restructures Net-Anionic PC/PG Monolayers with Saturated or Unsaturated Lipids. Langmuir 2016. Thurn, K. T.; Brown, E. M. B.; Wu, A.; Vogt, S.; Lai, B.; Maser, J.; Paunesku, T.; Woloschak, G. E. Nanoparticles for Applications in Cellular Imaging. Nanoscale Res. Lett. 2007, 2 (9), 430-441.

(28) You, S. S.; Rashkov, R.; Kanjanaboos, P.; Calderon, I.; Meron, M.; Jaeger, H. M.; Lin, B. Comparison of the Mechanical Properties of Self-Assembled Langmuir Monolayers of Nanoparticles and Phospholipids. Langmuir 2013, 29 (37), 11751-11757.

(29) Fang, C.; Bhattarai, N.; Sun, C.; Zhang, M. Functionalized Nanoparticles with Long-Term Stability in Biological Media. Small 209AD, 5 (14), 1637-1641.

(30) Malmsten, M.; Emoto, K.; Van Alstine, J. M. Effect of Chain Density on Inhibition of Protein Adsorption by Poly(ethylene Glycol) Based Coatings. J. Colloid Interface Sci. 1998, 202 (2), 507-517.

(31) Shkilnyy, A.; Soucé, M.; Dubois, P.; Warmont, F.; Saboungi, M.-L.; Chourpa, I. Poly(ethylene Glycol)Stabilized Silver Nanoparticles for Bioanalytical Applications of SERS Spectroscopy. Analyst 2009, 134 (9), $1868-1872$.

(32) Du, H.; Chandaroy, P.; Hui, S. W. Grafted Poly-(Ethylene Glycol) on Lipid Surfaces Inhibits Protein Adsorption and Cell Adhesion. Biochim. Biophys. Acta - Biomembr. 1997, 1326 (2), 236-248. 

and Flocculation Behavior of Polyethylene Glycol-Functionalized Silica Nanoparticles. J. Colloid Interface Sci. 2015, 452, 215-223.

(34) Noskov, B. A.; Akentiev, A. V; Alexandrov, D. A.; Miller, R. Dynamic Surface Elasticity of Aqueous Solutions of Polyethylene Glycol. Mendeleev Commun. 1998, 8 (5), 190-191.

(35) Sheffield, J. B. ImageJ, A Useful Tool for Biological Image Processing and Analysis. Microsc. Microanal. 2007, 13 (Suppl 2), 102-103.

(36) Azad, I.; Ram, M. K.; Goswami, D. Y.; Stefanakos, E. Fabrication and Characterization of ZnO LangmuirBlodgett Film and Its Use in Metal-Insulator-Metal Tunnel Diode. Langmuir 2016, 32 (33), 8307-8314.

(37) Sachan, A. K.; Harishchandra, R. K.; Bantz, C.; Maskos, M.; Reichelt, R.; Galla, H. J. High-Resolution Investigation of Nanoparticle Interaction with a Model Pulmonary Surfactant Monolayer. ACS Nano 2012, 6 (2), 1677-1687.

(38) Lee, K. Y. C. Collapse Mechanisms of Langmuir Monolayers. Annu. Rev. Phys. Chem. 2008, 59 (1), 771791.

(39) Vegso, K.; Siffalovic, P.; Majkova, E.; Jergel, M.; Benkovicova, M.; Kocsis, T.; Weis, M.; Luby, S.; Nygård, K.; Konovalov, O. Nonequilibrium Phases of Nanoparticle Langmuir Films. Langmuir 2012, 28, 10409-10414.

(40) Yi, P.; Chen, K. L. Influence of Solution Chemistry on the Release of Multiwalled Carbon Nanotubes from Silica Surfaces. Environ. Sci. Technol. 2013, 47 (21), 12211-12218. 
TOC Graphic
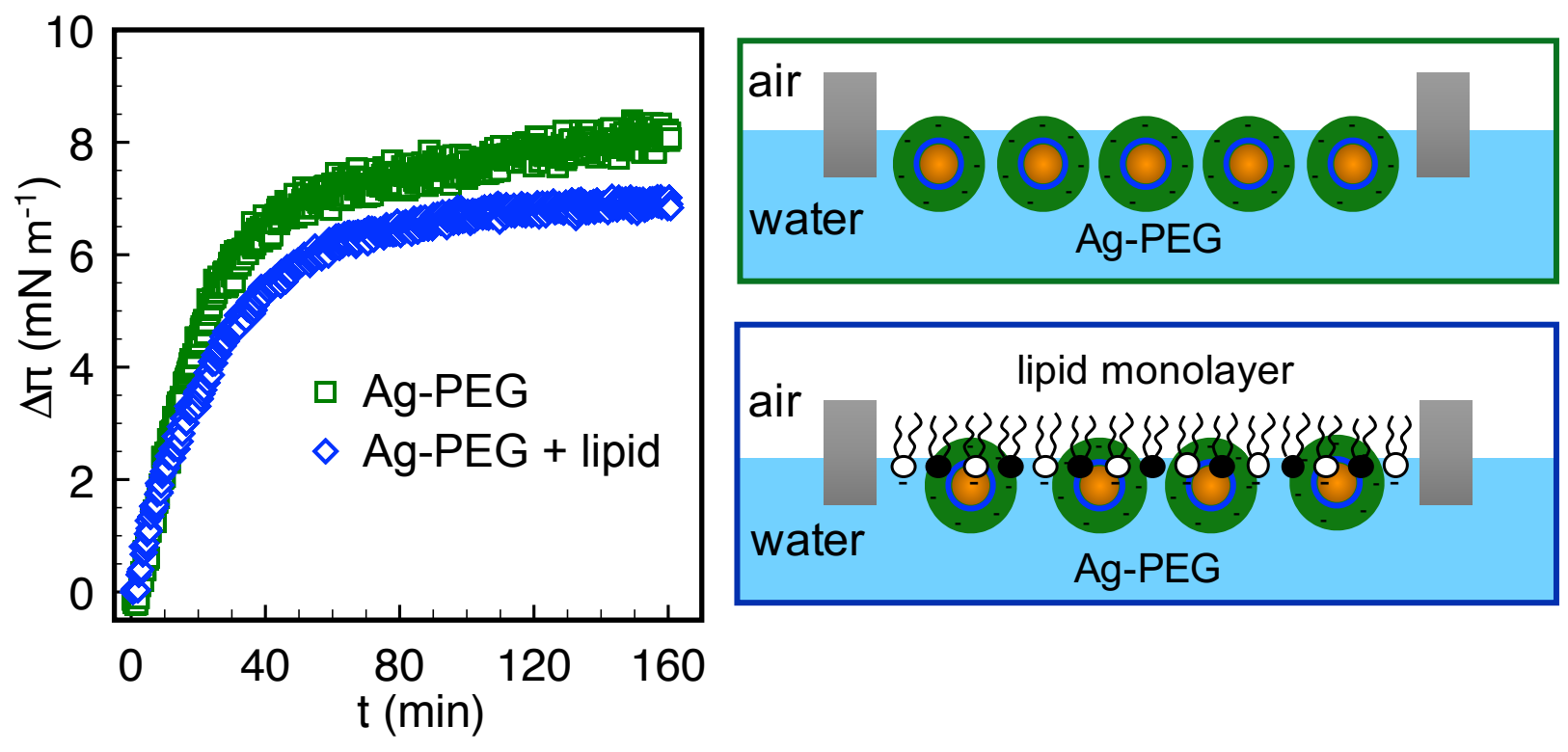